\section{EMERGENGY AND DISASTER MEDICINE IN THE MARYLAND SYSTEM}

\author{
C.R. LaMonica, R.N., Baltimore, \\ Maryland, U.S.A.
}

The purpose of this presentation is to provide an insight of how different health care facilities work, in conjunction with each other, to give efficient and appropriate care during emergency or disaster situations. In any disaster situation, whether natural, chemical, or social/civil unrest, it is essential that appropriate medical facilities function at their maximum capacity. Triage, started immediately by the field personnel, extends into the hospital and health care facilities in the surrounding areas of the disaster.

Through a network of Emergency Medical Service (EMS) personnel and hospital organizations, appropriate facilities are readily available at any time. The Maryland Institute for Emergency Medical Services Systems (MIEMSS) functions as a control center for the Maryland EMS system. Education and field training for emergency medical personnel, transportation and communication facilities, and improving treatment techniques, are managed by the MIEMSS-EMS coordinators. The hospital network in Maryland consists of multiple specialty referral centers. In the event of mass casualties, plans are designed to deliver an organized approach in total health care support to victims and communities.

\section{ROLE AND RESPONSIBILITY OF A POISON CENTER IN CHEMICAL ACCIDENTS}

\section{Per Kulling, M.D., Stockholm, Sweden}

A Poison Center has an important role in handling all types of chemical accidents where toxic effects on man can be expected by compiling information about the toxic agent including toxicity data, risks, first aid measures, hospital treatment, etc. and disseminating this information in the acute situation. Another important task for the Center in the chemical safety program is to take an active part in the contingency planning for chemical accidents and in education of rescue personnel and disaster planners. A Poison Center has a central position and can in this way act on a centralized basis for providing and adapting information for the rescue team, giving guidelines on supplying antidote stores, initiating collaboration between different institutions and organizations, etc.

Education must include lectures, training programs and exercises dealing with chemical accidents, and the Poison Center must take an active part in these activities. The rescue team must be provided with information about main risks, first aid measures, hospital treatment, etc. and this information must be adapted to these situations.

By initiating a close collaboration between different institutions and organizations like local hospitals, company health services, pathologists, Poison Centers, etc. much experience can be acquired from earlier accidents thus giving guidelines on preventive and therapeutic measures for the future. Designs for follow-up studies must be planned for.

A close collaboration between Poison Centers all over the world and between Poison Centers and other organizations dealing with disaster planning, like the World Association of Emergency and Disaster Medicine, The International Program on Chemical Safety, etc., is of course of vital importance.

In conclusion, the primary theme of disaster management is information management. Timely information can change the entire character of an accident and the Poison Center has a central position in this matter.

\section{THE MEDICAL DISASTER MANAGEMENT PLAN FOR THE ROTTERDAM HARBOR}

\author{
H.N. Hart, M.D., Ph.D., Rotterdam, \\ The Netherlands
}

The city of Rotterdam is intimately linked with the world's largest harbor, a complex with store facilities, industrial plants and oil refineries. A regional disaster management structure has been developed under the auspices of the Rotterdam Public Authority.

Initial action on the site of the accident is the responsibility of an alerted coordination team on the scene, consisting of representatives of the fire brigade, police, health service and port authority. The cooperation is optimalized through integrated training programs and regular exercises. An operations center is installed in one of the main buildings of the 
services involved and, whenever necessary, a management team is assembled in the town hall, supervised by the burgomaster.

Seventy municipal and private ambulances in the region can be mobilized through the regional dispatch center. This center is fully computerized. This system is capable of transporting 65 stretcher cases in the first hour and up to 100 stretcher cases in the second hour, before requesting additional assistance from neighboring regional dispatch centers.

If required, mobile hospital trauma teams will be made available. About 25 hospitals in the Netherlands are stand-by for producing such teams, which resort administratively under the Ministry of Welfare, Culture and Health. They can be used for first aid and triage on the scene or for rendering services to victims in neighboring hospitals. In connection with legally founded Municipal Disaster Management Plans, hospitals dispose of Hospital Disaster Management Plans, prescribing the number of victims to be admitted. The Red Cross is capable of setting up a casualty assembly center within about two hours of receiving the alert.

More details of the Medical Disaster Plan will be discussed.

\section{A SYSTEM FOR EMERGENCY MEDICAL RESPONSE TO UNDERGROUND DISASTERS}

\author{
Richard F. Kunkle, M.D., New Florence, \\ Pennsylvania, U.S.A.
}

A system has been developed by which physicians and paramedic based on-scene acute medical care may be delivered in the event of underground disasters. This system was designed to meet the needs of the deep mine industry in the eastern United States, but is appropriate to other locations and applications. The system is composed of residency trained Emergency Medicine physicians and specially trained and certified paramedics. These individuals are supplied with sufficient equipment and adequately trained to treat critical injuries insitu, either before or during prolonged extrication in confined or unusual locations. The purpose of the team is to reverse critical life-threatening injuries and prolong survivability of patients requiring extended extrication such as occurs during mine disasters or deep cave rescues.

The team may also be configured to respond as a self-contained rapid deployment unit capable of triaging and stabilizing 10 to 15 mixed category patients per hour for distribution to appropriate facilities. The team may be appropriately used as one component of the developing NDMS System (National Disaster Medical System). The team may also provide significant medical augmentation for other specialized rescue services such as trench rescue, wilderness rescue, etc.

Applicability of the team to related disasters such as earthquake response, building collapse and terrorist attack where prolonged extrication may significantly reduce patient survivability will be discussed. Current utilization of the team in other specialized situations will be mentioned. Composition of the team, training, equipment, financing, availability, historical responses, and potential for expansion of the concept will be reviewed. 